\title{
COVID-19 Vaccination Willingness among Chinese Adults under the Free Vaccination Policy
}

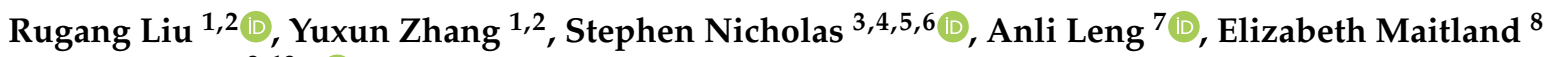 \\ and Jian Wang $9,10, *$ (D)
}

1 School of Health Policy \& Management, Nanjing Medical University, Nanjing 211166, China; rugangliu@njmu.edu.cn (R.L.); yuxunzhang@njmu.edu.cn (Y.Z.)

2 Center for Global Health, Nanjing Medical University, Nanjing 211166, China

3 Australian National Institute of Management and Commerce, Eveleigh, NSW 2015, Australia; stephen.nicholas@newcastle.edu.au

4 Research Institute for International Strategies, Guangdong University of Foreign Studies, Guangzhou 510420, China

5 School of Economics and School of Management, Tianjin Normal University, Tianjin 300074, China

6 Newcastle Business School, University of Newcastle, Newcastle, NSW 2308, Australia

7 School of Political Science and Public Administration, Institute of Governance, Shandong University, Qingdao 266237, China; lenganli@sdu.edu.cn

8 School of Management, University of Liverpool, Chatham Building, Chatham Street, Liverpool L69 7ZH, UK; e.maitland@liverpool.ac.uk

9 Dong Fureng Economic and Social Development School, Wuhan University, Wuhan 430072, China

10 Center for Health Economics and Management, School of Economics and Management, Wuhan University, Wuhan 430072, China

* Correspondence: wangjian993@whu.edu.cn

Citation: Liu, R.; Zhang, Y.; Nicholas, S.; Leng, A.; Maitland, E.; Wang, J. COVID-19 Vaccination Willingness among Chinese Adults under the Free Vaccination Policy. Vaccines 2021, 9, 292. https://doi.org/10.3390/ vaccines 9030292

Academic Editor: Martin H. Bluth

Received: 12 February 2021

Accepted: 17 March 2021

Published: 21 March 2021

Publisher's Note: MDPI stays neutral with regard to jurisdictional claims in published maps and institutional affiliations.

Copyright: (c) 2021 by the authors. Licensee MDPI, Basel, Switzerland This article is an open access article distributed under the terms and conditions of the Creative Commons Attribution (CC BY) license (https:/ / creativecommons.org/licenses/by/ $4.0 /)$.
Abstract: (1) Background: China will provide free coronavirus disease 2019 (COVID-19) vaccinations for the entire population. This study analyzed the COVID-19 vaccination willingness rate (VWR) and its determinants under China's free vaccination policy compared to a paid vaccine. (2) Methods: Data on 2377 respondents were collected through a nationwide questionnaire survey. Multivariate ordered logistic regression models were specified to explore the correlation between the VWR and its determinants. (3) Results: China's free vaccination policy for COVID-19 increased the VWR from $73.62 \%$ to $82.25 \%$ of the respondents. Concerns about the safety and side-effects were the primary reason for participants' unwillingness to be vaccinated against COVID-19. Age, medical insurance and vaccine safety were significant determinants of the COVID-19 VWR for both the paid and free vaccine. Income, occupation and vaccine effectiveness were significant determinants of the COVID-19 VWR for the free vaccine. (4) Conclusions: Free vaccinations increased the COVID-19 VWR significantly. People over the age of 58 and without medical insurance should be treated as the target intervention population for improving the COVID-19 VWR. Contrary to previous research, high-income groups and professional workers should be intervention targets to improve the COVID-19 VWR. Strengthening nationwide publicity and education on COVID-19 vaccine safety and effectiveness are recommended policies for decision-makers.

Keywords: COVID-19; vaccination willingness; free vaccination policy; determinant

\section{Introduction}

At the end of January 2021, the World Health Organization reported over 102.1 million infected cases, and over 2.2 million deaths, globally from the coronavirus disease 2019 (COVID-19) [1]. Vaccinations will be the most effective and economic way to prevent COVID-19 and control its spread [2], and people's vaccination willingness will decide whether they will receive the COVID-19 vaccine. During 2021, China's COVID-19 prevention and control policy [3] will provide free COVID-19 vaccinations for high-risk cohorts 
and then the general population. The government's free vaccination program, and the successful management of COVID-19, will depend on people's vaccination willingness. High vaccination rates protect both the vaccinated and unvaccinated, create herd immunity and reduce the risk of virus mutations. This study analyzed the determinants affecting people's COVID-19 vaccination willingness under China's free vaccination policy compared to the paid vaccine. The survey was conducted in May-June 2020 before the announcement on 9 January 2021 of China's free vaccination program.

Previous vaccination studies have shown that many factors are responsible for the COVID-19 vaccination willingness rate (VWR), such as socio-economic factors, awareness of the severity and susceptibility to the disease and trust in the vaccine [4-8]. From a survey in Japan, Yoda and Katsuyama found that males, older age people, rural residents and chronic disease sufferers displayed the highest willingness toward COVID-19 vaccination [4]. Marital status and trust in the health service system were found to be key determinants of COVID-19 vaccination willingness in Saudi Arabia [9]. Using an online survey of American adults, Reiter et al. found that participants were more likely to get vaccinated when they perceived a higher likelihood of getting a COVID-19 infection in the future, perceived a heightened severity of COVID-19 infection and perceived greater effectiveness in a COVID-19 vaccine; they were less likely to get vaccinated when they perceived higher potential vaccine harm [5]. Several studies have also been carried out on special populations, such as healthcare workers, long-term care staff and caregivers, which found that concerns about vaccine safety, side effects and effectiveness were the primary reasons for vaccine hesitancy $[7,10-13]$. A Chinese national online survey found that participants that perceived the benefits and were unconcerned about the efficacy of COVID-19 vaccines had the highest intention to vaccinate [6]. Their willingness-to-pay (WTP) for COVID-19 vaccines was influenced by social and demographic factors, such as occupation and region. A number of discrete choice experiments revealed that a strong preference for the COVID-19 vaccine depended on its effectiveness, side-effects, protection duration and number of injections [14-17]. However, little is currently known about the effect of free vaccinations on the COVID-19 VWR in China. To address this lacuna, we surveyed participants' (un)willingness to vaccinate when the vaccine was free; identified the reasons participants would get vaccinated or not; and recommend measures to improve the COVID-19 VWR.

\section{Materials and Methods}

\subsection{Data Source and Sample}

A questionnaire was designed to collect COVID-19 vaccination willingness and other variables. China's 27 provinces were divided into three regions: eastern, central and western. The provinces in each region were stratified into low, medium and high economic levels according to their 2019 gross domestic product (GDP). Randomly, one province was chosen from each economic level in each region, yielding nine provinces. Next, according to their 2019 GDP rank, all the cities in each selected province were divided into low, medium and high economic levels. One city was randomly chosen from each GDP level, with 27 cities selected from the 9 provinces. One hundred participants were interviewed faceto-face, or by online video interviews in cities where participants were required to home quarantine, in each city, with equal numbers of men and women and three urban residents for every two rural residents, which reflected the nationwide urban-rural breakdown. All investigators recruited in the 27 cities received standardized training before the formal investigations. During 30 May to 10 June 2020, face-to-face interviews were conducted by interviewers. All participants were informed about the purpose of the survey and gave informed consent. We collected data on 2700 adults over the age of 18 years old, which yielded a sample of 2377 respondents after deleting cases with missing data, with a response rate of $88.04 \%$. 


\subsection{Definition and Measurement of Dependent Variables}

The categorical dependent variable, COVID-19 vaccination willingness, was assessed by two questions: "Would you pay for the COVID-19 vaccination?" and "Would you get the COVID-19 vaccination if the vaccine were free?" There were three answers ("no" (0), "it depends" (1) and "yes" (2)) for each question, representing COVID-19 vaccination willingness being low, medium and high.

\subsection{Definition and Measurement of Independent Variables}

As shown in Table 1, the independent variables comprised sex (male-female), age groups, three average monthly income groups (low ( $<$ RMB4000), medium $(\geq$ RMB4000$<$ RMB8000) and high ( $\geq$ RMB8000)), education level (below high school, and high school and above), occupation, medical insurance (yes/no), urban-rural residence, self-rated health, residence in east-west-central region, and awareness of COVID-19 vaccine effectiveness, safety and risk of infection. Three urban residents were interviewed for every two rural participants. Occupations were categorized into professionals (including physicians, teachers and civil servants), farmers, students, self-employed, unemployed, migrant workers and other. Self-rated health was categorized into, "bad", "medium" and "good", based on the question: "How is your health status compared to your peers?" Participant's awareness of COVID-19 vaccine effectiveness and safety was measured by the questions: "Do you believe that the COVID-19 vaccine is effective?" /"Do you believe that the COVID-19 vaccine is safe?", and coded into a three-item Linkert scale ("don't agree-low effectiveness/safety", "neutral attitude-medium effectiveness/safety" and "agree-high effectiveness/safety"). The respondents' risk of infection was measured by asking participants whether they would be infected by COVID-19 in the future according to "low-neutral-high" measure of risk.

Table 1. Characteristic of respondents.

\begin{tabular}{|c|c|c|c|}
\hline \multicolumn{2}{|r|}{ Variables } & \multirow{2}{*}{$\begin{array}{c}\mathbf{N} \\
1154\end{array}$} & \multirow{2}{*}{$\begin{array}{c}\% \\
48.55\end{array}$} \\
\hline Sex & Male & & \\
\hline & Female & 1223 & 51.45 \\
\hline \multirow[t]{5}{*}{ Age } & $18-27$ & 915 & 38.49 \\
\hline & $28-37$ & 340 & 14.30 \\
\hline & $38-47$ & 460 & 19.35 \\
\hline & $48-57$ & 426 & 17.92 \\
\hline & $58+$ & 236 & 9.93 \\
\hline \multirow[t]{3}{*}{ Income } & High $(\geq$ RMB8000) & 813 & 34.20 \\
\hline & Medium ( $\geq$ RMB4000-<RMB8000) & 796 & 33.49 \\
\hline & Low $(<$ RMB4000) & 768 & 32.31 \\
\hline \multirow[t]{2}{*}{ Education level } & Below high school & 1472 & 61.93 \\
\hline & High school and above & 905 & 38.07 \\
\hline \multirow[t]{8}{*}{ Occupation } & Professional & 708 & 29.79 \\
\hline & Farmer & 278 & 11.70 \\
\hline & Migrant worker & 289 & 12.16 \\
\hline & Self-employed & 221 & 9.30 \\
\hline & Unemployed & 103 & 4.33 \\
\hline & Student & 639 & 26.88 \\
\hline & Retired & 86 & 3.62 \\
\hline & Other & 53 & 2.23 \\
\hline \multirow[t]{2}{*}{ Medical insurance } & Yes & 2289 & 96.30 \\
\hline & No & 88 & 3.70 \\
\hline \multirow[t]{2}{*}{ Residence } & Urban & 1462 & 61.51 \\
\hline & Rural & 915 & 38.49 \\
\hline \multirow[t]{3}{*}{ Self-rated health } & Bad & 73 & 3.07 \\
\hline & Medium & 564 & 23.73 \\
\hline & Good & 1740 & 73.20 \\
\hline
\end{tabular}


Table 1. Cont.

\begin{tabular}{|c|c|c|c|}
\hline \multicolumn{2}{|c|}{ Variables } & \multirow{2}{*}{$\frac{\mathbf{N}}{748}$} & \multirow{2}{*}{$\begin{array}{c}\% \\
31.47\end{array}$} \\
\hline Region & Eastern & & \\
\hline & Central & 686 & 28.86 \\
\hline & Western & 943 & 39.67 \\
\hline \multirow[t]{3}{*}{ Vaccine effectiveness } & Low & 17 & 0.72 \\
\hline & Medium & 301 & 12.66 \\
\hline & High & 2059 & 86.62 \\
\hline \multirow[t]{3}{*}{ Vaccine safety } & Low & 40 & 1.68 \\
\hline & Medium & 378 & 15.90 \\
\hline & High & 1959 & 82.41 \\
\hline \multirow[t]{3}{*}{ Risk of infection } & Low & 1107 & 46.57 \\
\hline & Medium & 670 & 28.19 \\
\hline & High & 600 & 25.24 \\
\hline
\end{tabular}

\subsection{Statistical Analyses}

All data were double-entered using EpiData 3.1 and checked for consistency. Statistical analyses were performed using STATA 12.0. The Pearson chi-square test was used to compare the differences in VWRs among different subgroups and the VWR differences between the paid and free vaccine. Multivariate ordered logistic regression models and odds ratio (OR) were used to assess the associations between each independent variable and the VWRs of the paid and free COVID-19 vaccines.

\section{Results}

\subsection{Characteristics of Respondents}

Table 1 shows the characteristics of 2377 survey respondents, with the male (49\%) and female (51\%) sex ratio broadly even and the urban (62\%)-rural (38\%) split close to the 3:2 national urban-rural ratio; the median age was 35; the median monthly income was RMB5000 and the three low-, medium- and high-income groups were roughly equal. In terms of education, 38.07\% respondents had a high school and above education level. Professionals (29.79\%) accounted for the highest occupational group, followed by students $(26.88 \%)$, migrant workers $(12.16 \%)$ and farmers $(11.70 \%)$. Only 3.07\% reported their selfassessed health as "bad" and $96 \%$ had one or more type of medical insurance. There was a broadly equal number of respondents from the eastern $(31.47 \%)$, central $(28.86 \%)$ and western (39.67\%) regions. Respondents mainly believed that the COVID-19 vaccine was effective $(86.62 \%) ; 82.41 \%$ believed that the vaccine was safe and $53.43 \%$ thought they were at medium or high risk of COVID-19 infection.

\subsection{COVID-19 Vaccination Willingness Rate}

Figure 1 shows that the COVID-19 VWR increased from $73.62 \%$ to $82.25 \%$ when vaccination changed from paid to free, and the uncertain group fell from $20.7 \%$ to $14.05 \%$ $\left(\mathrm{Chi}^{2}=827.89, p<0.001\right)$. For the paid vaccine, only $5.7 \%$ were unwilling to vaccinate: first, because of concerns about the safety and side-effects of the COVID-19 vaccine (32.99\%); second, because there was no perceived need for vaccination because the COVID-19 outbreak had been controlled in China (23.71\%); and third, because of the expense of the vaccine $(15.46 \%)$. For the free vaccine, only $3.7 \%$ were unwilling to vaccinate, with $51.32 \%$ worried about the safety and side-effects of COVID-19 vaccines; $13.16 \%$ did not believe that the vaccine was effective and $9.21 \%$ believed that they would not be infected in the future and that it was unnecessary to vaccinate against COVID-19.

\subsection{Results of Chi-Square Test}

Table 2 shows that the COVID-19 VWR for the free vaccine was higher than that of the paid vaccine in all subgroups $(p<0.05)$, except for the low vaccine effectiveness group, the VWR of which was $47.06 \%$ for the paid vaccine and $41.18 \%$ for the free vaccine $(p=0.005)$. For both the paid and free vaccine, the COVID-19 VWRs of the below high school education 
group was higher than the high school and above group $(p<0.05)$; farmers retained the highest VWR among all occupations, with a 76.62\% VWR for the paid vaccine and $88.85 \%$ VWR for the free vaccine ( $p=0.001)$; the VWR of respondents who had medical insurance was higher than that of non-insured respondents $(p<0.05)$; and the VWR increased with participants' increased awareness of vaccine effectiveness and safety $(p<0.001)$. The VWR of the 28-37 age group was the highest, and the VWR of the over-58 age group was the lowest for the paid vaccine group, with a VWR of $69.49 \%(p<0.001)$, but it rose to $83.9 \%$ for the free vaccine $(p=0.001)$. There were no differences among income groups for the paid vaccine, but the VWR decreased with income when the vaccine was free $(p=0.004)$, with the highest VWR for the low-income group (86.2\%). The central region had the highest VWR $(83.53 \%)$, followed by the western area $(82.61 \%)$ and the eastern area $(80.61 \%)$, for the free vaccine $(p=0.034)$. The participants with the highest awareness of a COVID-19 infection risk were most willing to get vaccinated, with a VWR of $84.33 \%$ for the free vaccine $(p=0.013)$.

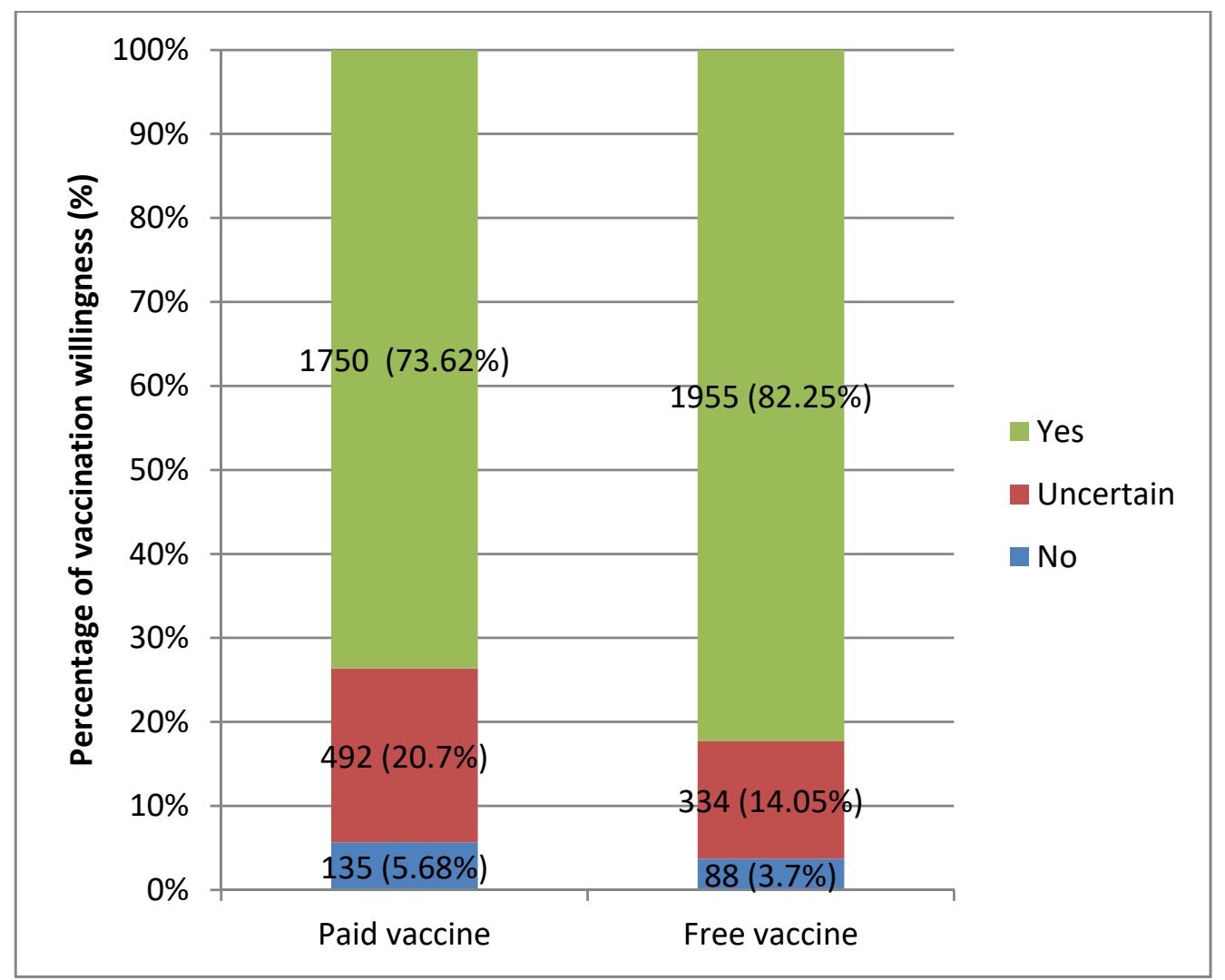

Figure 1. Comparison of COVID-19 vaccination willingness rates (VWRs) between paid and free vaccine.

\subsection{Results of Multiple Ordered Logistic Regressions}

Table 3 shows the results of two multivariate ordered logistic regression models specified to analyze the relationship between the VWR and the independent variables for the paid and free vaccine. Sex, education level, urban-rural residence, self-rated health, region and awareness of infection risk had no influence on VWR for both paid and free vaccines. The COVID-19 VWR of respondents aged above 58 was significantly lower than the 18-27 age group $(\mathrm{OR}=0.581, p=0.019$ for the paid vaccine; $\mathrm{OR}=0.456, p=0.006$ for the free vaccine) and respondents without medical insurance had a lower COVID-19 VWR than respondents who had medical insurance $(\mathrm{OR}=0.496, p=0.002$ for the paid vaccine; $\mathrm{OR}=0.513, p=0.011$ for the free vaccine). The COVID-19 VWR increased with vaccine safety for both the paid $(\mathrm{OR}=2.061, p=0.037$ (medium group); $\mathrm{OR}=4.692, p<0.001$ (high group) ) and free $(\mathrm{OR}=2.071, p=0.046$ (medium group); $\mathrm{OR}=6.641, p<0.001$ (high 
group)) vaccine. There was no difference in VWR among different income groups for the paid vaccine, but the COVID-19 VWR for the low-income group was higher than for highincome group for the free vaccine $(\mathrm{OR}=1.536, p=0.009)$. There was no statistical difference between the VWRs of famers and professionals for the paid vaccine, while the farmers had a higher VWR than professionals when the vaccine was free ( $\mathrm{OR}=2.016, p=0.008)$. Self-employed respondents had a lower VWR than professional participants for the paid vaccine (OR $=0.695, p=0.046)$, but there was no difference between the self-employed and professionals in the free vaccine model in Table 3. For the paid vaccine, there was no difference in the COVID-19 VWRs among respondents with different awareness levels of vaccine effectiveness; however, the VWR of people with a high awareness level of vaccine effectiveness was higher than the low awareness group ( $\mathrm{OR}=5.49, p=0.002)$.

Table 2. Comparison of COVID-19 vaccination willingness for paid and free vaccination with independent variables.

\begin{tabular}{|c|c|c|c|c|c|c|c|c|c|c|c|}
\hline \multicolumn{2}{|c|}{ Variables } & \multicolumn{4}{|c|}{ VWR of Paid Vaccine } & \multicolumn{4}{|c|}{ VWR of Free Vaccine } & \multicolumn{2}{|c|}{$\begin{array}{c}\text { Comparison } \\
\text { between Paid and } \\
\text { Free Vaccination }\end{array}$} \\
\hline & & $\mathbf{N}$ & $\%$ & $x^{2}$ & $p$ & $\mathbf{N}$ & $\%$ & $x^{2}$ & $p$ & $x^{2}$ & $p$ \\
\hline \multirow[t]{2}{*}{ Sex } & Male & 862 & 74.70 & 1.50 & 0.471 & 953 & 82.58 & 0.72 & 0.698 & 498.37 & $<0.001$ \\
\hline & Female & 888 & 72.61 & & & 1002 & 81.93 & & & 346.51 & $<0.001$ \\
\hline \multirow[t]{5}{*}{ Age } & $18-27$ & 670 & 73.22 & 63.75 & $<0.001$ & 755 & 82.51 & 25.12 & 0.001 & 244.42 & $<0.001$ \\
\hline & $28-37$ & 266 & 78.24 & & & 291 & 85.59 & & & 93.89 & $<0.001$ \\
\hline & $38-47$ & 321 & 69.78 & & & 362 & 78.70 & & & 185.32 & $<0.001$ \\
\hline & $48-57$ & 329 & 77.23 & & & 349 & 81.92 & & & 208.45 & $<0.001$ \\
\hline & $58+$ & 164 & 69.49 & & & 198 & 83.90 & & & 66.22 & $<0.001$ \\
\hline \multirow[t]{3}{*}{ Income } & High & 602 & 74.05 & 2.95 & 0.565 & 642 & 78.97 & 15.35 & 0.004 & 339.79 & $<0.001$ \\
\hline & Medium & 584 & 73.37 & & & 651 & 81.78 & & & 271.24 & $<0.001$ \\
\hline & Low & 564 & 73.44 & & & 662 & 86.20 & & & 235.30 & $<0.001$ \\
\hline \multirow[t]{2}{*}{ Education level } & Below high school & 1093 & 74.25 & 22.71 & $<0.001$ & 1228 & 83.42 & 7.68 & 0.021 & 536.48 & $<0.001$ \\
\hline & High school and above & 657 & 72.60 & & & 727 & 80.33 & & & 280.16 & $<0.001$ \\
\hline \multirow[t]{8}{*}{ Occupation } & Professional & 525 & 74.15 & 36.93 & 0.001 & 559 & 78.95 & 35.57 & 0.001 & 290.55 & $<0.001$ \\
\hline & Farmer & 213 & 76.62 & & & 247 & 88.85 & & & 99.27 & $<0.001$ \\
\hline & Migrant worker & 220 & 76.12 & & & 239 & 82.70 & & & 104.51 & $<0.001$ \\
\hline & Self-employed & 152 & 68.78 & & & 180 & 81.45 & & & 78.91 & $<0.001$ \\
\hline & Unemployed & 71 & 68.93 & & & 89 & 86.41 & & & 29.02 & $<0.001$ \\
\hline & Student & 468 & 73.24 & & & 525 & 82.16 & & & 184.11 & $<0.001$ \\
\hline & Retired & 63 & 73.26 & & & 73 & 84.88 & & & 43.66 & $<0.001$ \\
\hline & Other & 38 & 71.70 & & & 43 & 81.13 & & & 13.47 & 0.009 \\
\hline \multirow[t]{2}{*}{ Medical insurance } & Yes & 1700 & 74.27 & 13.66 & 0.001 & 1892 & 82.66 & 8.39 & 0.015 & 786.92 & $<0.001$ \\
\hline & No & 50 & 56.82 & & & 63 & 71.59 & & & 36.37 & $<0.001$ \\
\hline \multirow[t]{2}{*}{ Residence } & Urban & 1080 & 73.87 & 0.84 & 0.657 & 1197 & 81.87 & 0.39 & 0.821 & 582.10 & $<0.001$ \\
\hline & Rural & 670 & 73.22 & & & 758 & 82.84 & & & 258.51 & $<0.001$ \\
\hline \multirow[t]{3}{*}{ Self-rated health } & Bad & 50 & 68.49 & 7.79 & 0.100 & 60 & 82.19 & 1.17 & 0.883 & 11.14 & 0.025 \\
\hline & Medium & 400 & 70.92 & & & 466 & 82.62 & & & 161.68 & $<0.001$ \\
\hline & Good & 1300 & 74.71 & & & 1429 & 82.13 & & & 680.67 & $<0.001$ \\
\hline \multirow[t]{3}{*}{ Region } & Eastern & 546 & 72.99 & 6.80 & 0.147 & 603 & 80.61 & 10.40 & 0.034 & 265.17 & $<0.001$ \\
\hline & Central & 505 & 73.62 & & & 573 & 83.53 & & & 386.46 & $<0.001$ \\
\hline & Western & 699 & 74.13 & & & 779 & 82.61 & & & 188.60 & $<0.001$ \\
\hline \multirow[t]{3}{*}{ Vaccine effectiveness } & Low & 8 & 47.06 & 124.14 & $<0.001$ & 7 & 41.18 & 245.71 & $<0.001$ & 14.85 & 0.005 \\
\hline & Medium & 147 & 48.84 & & & 162 & 53.82 & & & 138.87 & $<0.001$ \\
\hline & High & 1595 & 77.46 & & & 1786 & 86.74 & & & 529.37 & $<0.001$ \\
\hline \multirow[t]{3}{*}{ Vaccine safety } & Low & 18 & 45.00 & 167.88 & $<0.001$ & 21 & 52.50 & 279.62 & $<0.001$ & 25.40 & $<0.001$ \\
\hline & Medium & 194 & 51.32 & & & 214 & 56.61 & & & 199.46 & $<0.001$ \\
\hline & High & 1538 & 78.51 & & & 1720 & 87.80 & & & 421.77 & $<0.001$ \\
\hline \multirow[t]{3}{*}{ Risk of infection } & Low & 802 & 72.45 & 9.22 & 0.056 & 914 & 82.57 & 12.69 & 0.013 & 394.98 & $<0.001$ \\
\hline & Medium & 485 & 72.39 & & & 535 & 79.85 & & & 250.14 & $<0.001$ \\
\hline & High & 463 & 77.17 & & & 506 & 84.33 & & & 206.12 & $<0.001$ \\
\hline
\end{tabular}


Table 3. Results of multiple ordered logistic regressions.

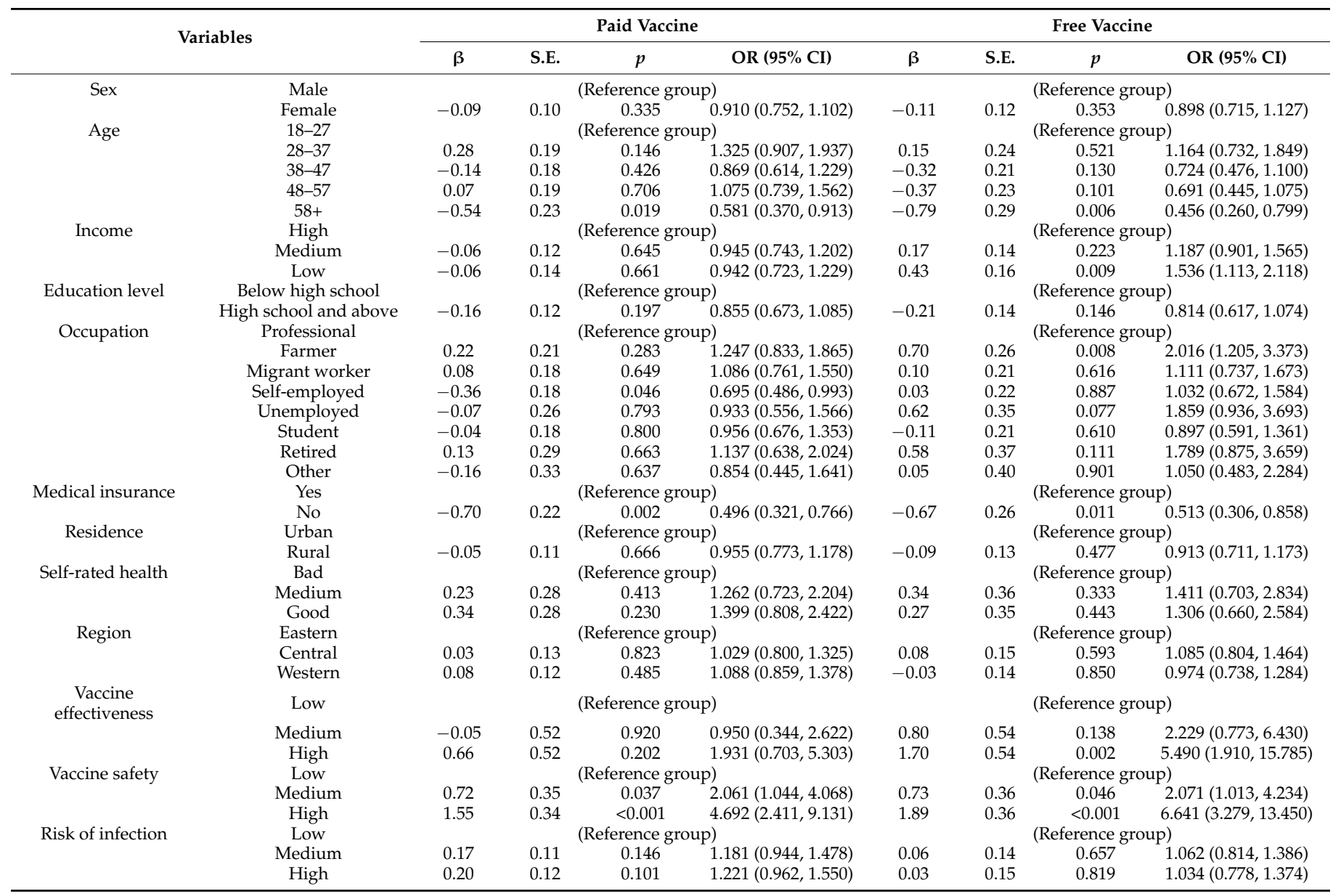

\section{Discussion}

The COVID-19 VWR and its influencing factors among Chinese adults were analyzed for a paid and a free vaccine. The outcomes can be used to guide projections of future COVID-19 vaccine uptake and shape vaccination policy. The COVID-19 VWR was $82.25 \%$ for the free vaccine, significantly higher than $73.62 \%$ for the paid vaccine, increasing the COVID-19 VWR by $11.72 \%$. For the paid vaccine, the VWR in this study was higher than in similar studies in Japan (65.7\%) [4], France (62\%), Germany (70\%) [18] and the United States (69\%) [5], but roughly the same as the Netherlands (73\%), Italy (74\%) and Portugal (75\%); the VWR for the free vaccine was similar to Denmark (80\%) [18] and Australia (85.8\%) [19].

Age, medical insurance and vaccine safety were significant influencing factors in both the paid and free vaccine regression models; sex, education level, urban-rural residence, self-rated health, region and awareness of infection risk were not significant factors in VWR. Although free vaccinations raised COVID-19 VWR from $69.49 \%$ to $83.9 \%$ for respondents aged over 58 years old, their VWR remained lower than the 18-27 age group for both paid $(\mathrm{OR}=0.581)$ and free vaccinations $(\mathrm{OR}=0.456)$. This finding was counter to previous studies in Japan and the United States, which showed that the elderly had a higher COVID19 vaccination willingness $[4,20]$. The COVID-19 VWR of people who had no medical insurance was only half that of those who had medical insurance $(\mathrm{OR}=0.496$ for the paid vaccine, $\mathrm{OR}=0.513$ for the free vaccine) even through the COVID-19 VWR of people without medical insurance increased from $56.82 \%$ to $71.59 \%$ when the vaccine was free. This finding is similar to past research, which found the COVID-19 vaccine acceptance of people with private or public health insurance was higher than people without health insurance [5]. One recommendation from our study is that special measures should be 
taken among people aged over 58 years old and without medical insurance to enhance their VWR.

Vaccine safety had the greatest impact on COVID-19 VWR, with the biggest OR $=6.63$ for the high vaccine safety group in the free vaccine model. The COVID-19 VWR increased when respondents' awareness of vaccine safety increased, consistent with a U.S. study that found that perceptions of the potential harm of a COVID-19 vaccine decreased people's willingness to get vaccinated. However, the U.S. study also found that perceiving a risk of getting a COVID-19 infection in the future increased the VWR, which was different from the result in our study [5]. A study in Kuwait also showed that the respondents were less willing to accept COVID-19 vaccination when they viewed vaccines in general to have health-related risks [21]. We suggest that improving people's awareness of vaccine safety will help to increase the COVID-19 VWR.

Income had no influence on COVID-19 VWR in the paid vaccine model, but the low-income group had a higher COVID-19 VWR than the high-income group in the free vaccine model $(86.2 \%$ vs. $78.97 \%$, OR $=1.536)$, which was different from previous research [5,6,22-25]. The VWR growth rate of free vaccinations for the low-income group was $17.37 \%$ (from $73.44 \%$ to $86.2 \%$ ), but that for the high-income group was only $6.64 \%$ (from $74.05 \%$ to $78.97 \%$ ). Free vaccinations had the greatest effect on the COVID-19 VWR of low-income people among all income groups. Surprisingly, one recommendation from our study is that new interventions should target the high-income group because their COVID-19 VWR was lower than the low-income group when vaccinations were free.

The COVID-19 VWR of self-employed people was lower than professionals for the paid vaccine. Famers' COVID-19 VWR increased from $76.62 \%$ to $88.85 \%$ with a growth rate of $15.96 \%$, and that of self-employed people increased from $68.78 \%$ to $81.45 \%$ when the vaccine was free. The COVID-19 VWR of professionals only increased from $74.15 \%$ to $78.95 \%$, with a growth rate of $6.47 \%$, and their VWR was also lower than famers' VWR for the free vaccine. While the self-employed should be a key intervention target for the paid vaccine, professionals are a key intervention group for free vaccinations.

For the paid vaccine, people's COVID-19 VWR was only affected by vaccine safety, without any influence from vaccine effectiveness in the regression analysis. For the free vaccine, people cared not only about vaccine safety, but also about vaccine effectiveness. These results are similar to previous studies that showed that the perceived effectiveness of a COVID-19 vaccine improved the VWR $[5,6,14,15,26]$. Worrying about the safety and side-effects was also a main reason for people's unwillingness to be vaccinated for both the paid vaccine (32.99\%) and free vaccine (51.32\%). Suspecting the effectiveness of vaccines was the second reason for unwillingness to take up the free vaccine $(13.16 \%)$ in this study. For both the paid and free vaccines, information and education campaigns should be launched to reassure the public that listed COVID-19 vaccines are safe and effective and thus enhance the public's trust in the COVID-19 vaccines [18,27-30]. Since urban-rural residence and regional location were not significant determinants of the VWR, information and education campaigns can be national.

\section{Strengths and Limitations}

This study has several strengths. First, this was the first study to assess the effect of a COVID-19 free vaccination policy on the COVID-19 VWR among Chinese adults. Second, the dataset came from a nationwide questionnaire survey, covering both rural and urban areas, and three (eastern, central and western) regions in China. Third, face-to-face or online video interviews were conducted to collect the data in contrast to most past research, which relied on online questionnaire surveys.

There were two major limitations. First, we used only one question to evaluate respondents' COVID-19 vaccination willingness; a more complex vaccination willingness scale should be developed in further studies. Second, the sample was limited to only 100 respondents in each city across three regions in China. 


\section{Conclusions}

Free vaccinations increased COVID-19 VWR by $11.72 \%$, increasing the willingness to vaccinate from $73.62 \%$ for the paid vaccine to $82.25 \%$ for the free vaccine. Given the negative health and economic impact of the COVID-19 virus, increased vaccination rates promotes herd immunity and China's return to pre-COVDI-19 normality. For both the paid and free vaccine, vaccine safety was the most significant concern among the study participants. The COVID-19 VWR for the free vaccine for respondents aged over 58 years old and without medical insurance was lower than the reference groups. Our results suggest that intervention measures should be directed towards people aged over 58 years old and without medical insurance to improve their COVID-19 VWR. For the free vaccine, the COVID-19 VWR of the high-income group was lower than the low-income group and that of professionals lower than farmers. Surprisingly, our results suggest that high-income groups and professionals should be special COVID-19 VWR intervention targets for the free vaccine. Strengthening COVID-19 vaccine knowledge through public information and education campaigns on vaccine safety would improve VWR, whether the vaccine is free or not. Vaccine effectiveness should be included in a nationwide COVID-19 education campaign, especially for the free vaccine.

Author Contributions: Conceptualization, R.L. and A.L. and J.W.; methodology, R.L. and Y.Z; software, R.L. and Y.Z; ; validation, S.N. and J.W. and R.L.; formal analysis, R.L. and Y.Z.; investigation, R.L. and A.L. and Y.Z.; resources, J.W. and A.L. and R.L.; data curation, R.L. and Y.Z.; writingoriginal draft preparation, R.L. and J.W. and Y.Z.; writing-review and editing, S.N. and E.M. and J.W.; supervision, J.W.; project administration, J.W. and A.L. and R.L.; funding acquisition, R.L. and A.L. All authors have read and agreed to the published version of the manuscript.

Funding: This research was funded by National Natural Science Foundation of China, grant number 71904089; the NHC Key Laboratory of Health Economics and Policy Research (Shandong University), grant number: NHC-HEPR2019006; the China Postdoctoral Science Foundation, grant number 2019M662392; and the Qingdao Postdoctoral Foundation.

Institutional Review Board Statement: The study was conducted according to the guidelines of the Declaration of Helsinki, and approved by the Ethics Committee of Nanjing Medical University (protocol code Nanjing Medical University Ethical Audit (2020) Number 565 and 16 June 2020).

Informed Consent Statement: Informed consent was obtained from all subjects involved in the study.

Data Availability Statement: The data presented in this study are available on request from the corresponding author. The data are not publicly available due to multi-cooperation with Wuhan University, Shandong University and Nanjing Medical University. The corresponding author will facilitate a discussion with these three universities for data access on a reasonable request.

Acknowledgments: The authors thank reviewers for their constructive comments and thank all interviewers from the nine sample provinces for their contribution to the survey.

Conflicts of Interest: The authors declare no conflict of interest.

\section{References}

1. Coronavirus Disease (COVID-2019) Situation Reports. Available online: https://www.who.int/emergencies/diseases/novelcoronavirus-2019/situation-reports (accessed on 4 February 2021).

2. DRAFT Landscape of COVID-19 Candidate Vaccines-30 April 2020. Available online: https://www.who.int/whodocumentsdetail/draft-landscape-of-COVID-19-candidate-vaccines (accessed on 16 March 2021).

3. COVID-19 Vaccination Free to Chinese Residents: Official. Available online: http://www.ecns.cn/news/2021-01-09/detailihafqmaz1834647.shtml (accessed on 5 February 2021).

4. Yoda, T.; Katsuyama, H. Willingness to Receive COVID-19 Vaccination in Japan. Vaccines 2021, 9, 48. [CrossRef]

5. Reiter, P.L.; Pennell, M.L.; Katz, M.L. Acceptability of a COVID-19 vaccine among adults in the United States: How many people would get vaccinated? Vaccine 2020, 38, 6500-6507. [CrossRef] [PubMed]

6. Lin, Y.; Hu, Z.; Zhao, Q.; Alias, H.; Danaee, M.; Wong, L.P. Understanding COVID-19 vaccine demand and hesitancy: A nationwide online survey in China. PLoS Negl. Trop. Dis. 2020, 14, e0008961. [CrossRef] 
7. Nzaji, M.K.; Ngombe, L.K.; Mwamba, G.N.; Ndala, D.B.B.; Miema, J.M.; Lungoyo, C.L.; Mwimba, B.L.; Bene, A.C.M.; Musenga, E.M. Acceptability of Vaccination Against COVID-19 Among Healthcare Workers in the Democratic Republic of the Congo. Pragm. Observ. Res. 2020, 11, 103-109. [CrossRef]

8. Graffigna, G.; Palamenghi, L.; Boccia, S.; Barello, S. Relationship between Citizens' Health Engagement and Intention to Take the COVID-19 Vaccine in Italy: A Mediation Analysis. Vaccines 2020, 8, 576. [CrossRef]

9. Mohammed, A.-M.; Kumar, P.B. Determinants of COVID-19 Vaccine Acceptance in Saudi Arabia: A Web-Based National Survey. J. Multidiscip. Healthc. 2020, 13, 1657-1663. [CrossRef]

10. Goldman, R.D.; Yan, T.D.; Seiler, M.; Cotanda, C.P.; Brown, J.C.; Klein, E.J.; Hoeffe, J.; Gelernter, R.; Hall, J.E.; Davis, A.L.; et al. International COVID-19 Parental Attitude Study Group Caregiver willingness to vaccinate their children against COVID-19: Cross sectional survey. Vaccine 2020. [CrossRef] [PubMed]

11. Verger, P.; Scronias, D.; Dauby, N.; Adedzi, K.A.; Gobert, C.; Bergeat, M.; Gagneur, A.; Dubé, E. Attitudes of healthcare workers towards COVID-19 vaccination: A survey in France and French-speaking parts of Belgium and Canada, 2020. Euro Surv. Bull. Eur. Mal. Trans. Eur. Commun. Dis. Bull. 2021, 26. [CrossRef]

12. Unroe, K.T.; Evans, R.; Weaver, L.; Rusyniak, D.; Blackburn, J. Willingness of long-term care staff to receive a COVID-19 vaccine: A single state survey. J. Am. Geriat. Soc. 2020. [CrossRef]

13. Bell, S.; Clarke, R.; Mounier-Jack, S.; Walker, J.L.; Paterson, P. Parents' and guardians' views on the acceptability of a future COVID-19 vaccine: A multi-methods study in England. Vaccine 2020. [CrossRef]

14. Leng, A.; Maitland, E.; Wang, S.; Nicholas, S.; Liu, R.; Wang, J. Individual preferences for COVID-19 vaccination in China. Vaccine 2021, 39, 247-254. [CrossRef]

15. Dong, D.; Xu, R.H.; Wong, E.L.-Y.; Hung, C.-T.; Feng, D.; Feng, Z.; Yeoh, E.-K.; Wong, S.Y.-S. Public preference for COVID-19 vaccines in China: A discrete choice experiment. Health Expect. Int. J. Public Particip. Health Care Health Policy 2020. [CrossRef]

16. Borriello, A.; Master, D.; Pellegrini, A.; Rose, J.M. Preferences for a COVID-19 vaccine in Australia. Vaccine 2020, 39, 473-479. [CrossRef] [PubMed]

17. García, L.Y.; Cerda, A.A. Contingent assessment of the COVID-19 vaccine. Vaccine 2020, 38, 5424-5429. [CrossRef]

18. Neumann-Böhme, S.; Varghese, N.E.; Sabat, I.; Barros, P.P.; Brouwer, W.; Exel, J.v.; Schreyögg, J.; Stargardt, T. Once we have it, will we use it? A European survey on willingness to be vaccinated against COVID-19. Eur. J. Health Econ. 2020, 21, 977-982. [CrossRef] [PubMed]

19. Dodd, R.H.; Cvejic, E.; Bonner, C.; Pickles, K.; McCaffery, K.J. Willingness to vaccinate against COVID-19 in Australia. Lancet Infect. Dis. 2020. [CrossRef]

20. Malik, A.A.; McFadden, S.M.; Elharake, J.; Omer, S.B. Determinants of COVID-19 vaccine acceptance in the US. EClinMed 2020, 26, 100495. [CrossRef]

21. Alqudeimat, Y.; Alenezi, D.; AlHajri, B.; Alfouzan, H.; Almokhaizeem, Z.; Altamimi, S.; Almansouri, W.; Alzalzalah, S.; Ziyab, A. Acceptance of a COVID-19 Vaccine and its Related Determinants among the General Adult Population in Kuwait. Med. Princ. Pract. Int. J. Kuwait Univ. Health Sci. Cent. 2021. [CrossRef]

22. Sarasty, O.; Carpio, C.E.; Hudson, D.; Guerrero-Ochoa, P.A.; Borja, I. The demand for a COVID-19 vaccine in Ecuador. Vaccine 2020, 38, 8090-8098. [CrossRef]

23. Willingness to pay for a COVID-19 vaccine high in Chile. Pharm. Econ. Outcomes News 2020, 858, 35. [CrossRef]

24. Harapan, H.; Wagner, A.L.; Yufika, A.; Winardi, W.; Anwar, S.; Gan, A.K.; Setiawan, A.M.; Rajamoorthy, Y.; Sofyan, H.; Vo, T.Q.; et al. Willingness-to-pay for a COVID-19 vaccine and its associated determinants in Indonesia. Hum. Vaccines Immunother. 2020, 16, 3074-3080. [CrossRef]

25. Wong, L.P.; Alias, H.; Wong, P.F.; Lee, H.Y.; AbuBakar, S. The use of the health belief model to assess predictors of intent to receive the COVID-19 vaccine and willingness to pay. Hum. Vaccines Immunother. 2020, 16, 2204-2214. [CrossRef] [PubMed]

26. Guidry, J.P.D.; Laestadius, L.I.; Vraga, E.K.; Miller, C.A.; Perrin, P.B.; Burton, C.W.; Ryan, M.; Fuemmeler, B.F.; Carlyle, K.E. Willingness to get the COVID-19 Vaccine with and without Emergency Use Authorization. Am. J. Infect. Control 2020, 49, 137-142. [CrossRef] [PubMed]

27. Jiang, S. Don't rush to deploy COVID-19 vaccines and drugs without sufficient safety guarantees. Nature 2020. [CrossRef] [PubMed]

28. Ehde, D.M.; Roberts, M.K.; Herring, T.E.; Alschuler, K.N. Willingness to obtain COVID-19 vaccination in adults with multiple sclerosis in the United States. Mult. Scler. Relat. Disord. 2021, 49, 102788. [CrossRef] [PubMed]

29. Miyachi, T.; Takita, M.; Senoo, Y.; Yamamoto, K. Lower trust in national government links to no history of vaccination. Lancet 2020, 395, 31-32. [CrossRef]

30. Liu, Z.; Yang, J.Z. In the Wake of Scandals: How Media Use and Social Trust Influence Risk Perception and Vaccination Intention among Chinese Parents. Health Commun. 2020, 1-12. [CrossRef] [PubMed] 